\title{
Evaluation of Candida bloodstream infection and antifungal utilization in a tertiary care hospital
}

\author{
Tatiana Aporta Marins ${ }^{1 *}$, Alexandre R. Marra ${ }^{2,3}$, Michael B. Edmond ${ }^{3,4}$, Marines Dalla Valle Martino ${ }^{5}$, \\ Paula Kiyomi Onaga Yokota ${ }^{2}$, Ana Carolina Cintra Nunes Mafra ${ }^{6}$ and Marcelino Souza Durão Junior ${ }^{2}$
}

\begin{abstract}
Background: Candida bloodstream infections carry a significant mortality risk, justifying the importance of adequate antifungal therapy. This study describes trends in antifungal consumption using the Defined Daily Dose (DDD) and Days of Therapy (DOT) metrics, identifies the microbiological profile, the time to initiation of empirical therapy, the adjustment after positive blood culture results for Candida, and the impact on in-hospital mortality rate in patients with candidemia.
\end{abstract}

Methods: An analysis of antifungal consumption from 2008 to 2016, and of candidemia cases from 2012 to 2016 was carried out in a private tertiary hospital.

Results: A total of 11,273 admissions were identified with a prescription for at least one type of antifungal therapy. Fluconazole was the most prescribed antifungal drug in terms of general consumption. Through the DDD and DOT metrics, we observed that over time, there was an increase in the consumption of liposomal amphotericin B, micafungin and voriconazole. Candida albicans was the most isolated species in blood cultures. Regarding candidemia, we analyzed samples from 115 patients. Empirical therapy was started within $24 \mathrm{~h}$ of blood culture in $44.3 \%$ of the cases, and in $81.7 \%$ of the cases, the antifungal was deemed to be adequate based in antifungal susceptibility testing, both of which were not associated with the in-hospital mortality rate.

Conclusions: Our study reinforces the importance of monitoring the consumption of antifungal agents, which helps in proposing actions that lead to their rational use and, consequently, reduces the appearance of resistant strains.

Keywords: Antifungal, Candidemia, Consumption, Defined daily dose, Days of therapy

\section{Background}

Since the 1980s, candidemia cases have increased substantially in different regions of the world and, in Brazilian hospitals, it is one of the highest incidence infections [1, 2]. In a recently published Brazilian study, Candida spp. was the seventh most prevalent agent in analyzed bloodstream infections [3]. Candida albicans remains the most commonly isolated microorganism among Candida, however, candidemia caused by non-albicans species has increased worldwide, particularly C. tropicalis, C. glabrata, C. parapsilosis and C. krusei $[4,5]$.

\footnotetext{
* Correspondence: tatiana.marins@einstein.br

${ }^{1}$ Hospital Israelita Albert Einstein, São Paulo, Brazil

Full list of author information is available at the end of the article
}

The severity of these infections poses a significant morbidity and mortality risk [6]. They are often expensive and difficult to treat, so adequate antifungal therapy is necessary [7-10]. Regarding the timing of initiation of empiric treatment, studies are still controversial, with some showing that mortality was lower in patients who received the antifungal early $[10,11]$ and others indicating that there was no significant difference in in-hospital mortality $[12,13]$.

In order to provide adequate antifungal therapy, it is important to know the consumption pattern and the microbiological profile of your institution and, with the obtained information, to implement opportunities for improvement that lead to the rational use of these drugs

(C) The Author(s). 2018 Open Access This article is distributed under the terms of the Creative Commons Attribution 4.0 International License (http://creativecommons.org/licenses/by/4.0/), which permits unrestricted use, distribution, and 
and consequently achieve therapeutic success and increase patient safety.

Despite the advances in antifungal development in the previous decade, the therapeutic arsenal available on the market, and validated for the treatment of systemic fungal infections, is still limited and candidemia and other forms of invasive candidiasis remain potentially fatal infections, justifying the careful choice of treatments [14, 15]. Currently, there are four classes of systemic antifungal drugs that are effective: polyenes, azoles, echinocandins, and flucytosine [16]. Consumption of these drugs should be monitored over the years to control overuse, measure improvements, and obtain parameters for future internal and external comparisons. Monitoring is also important to prevent resistant microorganism species in the future.

This monitoring should be performed through quantitative analysis $[17,18]$. There are already well established metrics for the consumption of antimicrobials, the most common being the Defined Daily Dose (DDD), but there are others such as: days of therapy (DOT), Prescribed Daily Dose (PDD), and Recommended Daily Dose (RDD), which are also being used for the calculation of antifungal consumption [19-22]. There is still no consensus as to what is the best metric to quantify the use of these drugs. The World Health Organization (WHO) recommends consumption calculation using DDD [22], but other authors suggest DOT, since this methodology is not influenced by changes in the recommended individual dose and by dose adjustment in cases of kidney failure $[23,24]$.

The goal of this study is to describe and analyze antifungal consumption trends through the DDD and DOT metrics in patients who were admitted to Hospital Israelita Albert Einstein, and also identify the microbiological profile, time of onset of empirical antifungal therapy, antifungal adequacy and the impact on in-hospital mortality in patients with candidemia.

\section{Methods}

The study was conducted in a 670-bed private, tertiary hospital focused on high complexity treatments including solid organ and bone marrow transplantation, located in the city of São Paulo, Brazil. This is a descriptive and retrospective observational study that identified the global consumption of antifungal drugs in all the patients who were hospitalized from January 1, 2008 to December 31, 2016. Adult inpatients with candidemia from January 1, 2012 to December 31, 2016 were also analyzed. This study was submitted and approved by the Institution's Research Ethics Committee and informed consent was not required.

The report of inpatient antifungal medicines was generated through the hospital's electronic inventory management system. The analyzed drugs were: fluconazole, voriconazole, itraconazole, posaconazole, caspofungin, micafungin, anidulafungin, liposomal amphotericin $\mathrm{B}$ and amphotericin B deoxycholate. Hospital nursing units include Adult Medical and Surgical Wards (MSW, $n$ = 14), Oncology (ONCO), Bone Marrow Transplantation (BMT), Solid Organ Transplantation (SOT), Adult Intensive Care Unit (AICU), Pediatrics (PED) and Neonatal Intensive Care Unit (NICU).

The impact of bed occupancy variation was minimized by reporting the antifungal consumption per 1000 patient-days. Two consumption metrics were used, DDD and DOT. We chose these metrics because they are the most commonly used and are recommended by health agencies [20, 22].

DDD is defined as the mean maintenance dose per day for a drug used in its primary indication in an adult patient [22]. The DOT metric measures the number of days that a patient is on a specific drug, regardless of the number of doses administered or dosing [25]. One DOT is assigned for each drug administered each day. To assess consumption in Pediatrics and Neonatology, only the DOT was calculated. DDD and DOT were calculated annually for general consumption data, and data by specialty. The formulas used for the DDD and DOT calculations were:

$$
\begin{aligned}
& \text { DDD } 1000 \text { patient-days } \\
& =\frac{\text { drug consumption in grams for period } \times 1000}{\text { IDD } \times \text { number of patient-days in the period }}
\end{aligned}
$$

DOT 1000 patient-days

$=\frac{\text { days of therapy in the period } \times 1000}{\text { number of patient-days in the period }}$

For the DDD calculation, the anatomical therapeutic chemical (ATC)/DDD system, recognized by the World Health Organization as an international standard for drug use studies, was used. The Defined Daily Doses used were $200 \mathrm{mg}$ for fluconazole, $400 \mathrm{mg}$ for voriconazole, $200 \mathrm{mg}$ for itraconazole, $300 \mathrm{mg}$ for posaconazole, $50 \mathrm{mg}$ for caspofungin, $100 \mathrm{mg}$ for micafungin, $100 \mathrm{mg}$ for anidulafungin and $35 \mathrm{mg}$ for amphotericin B deoxycholate and liposomal amphotericin B [22].

In analyzing the medical records for hospitalized patients diagnosed with candidemia in the period between 2012 and 2016, patients under the age of 18 years, outpatients and those who had incomplete data in their medical records were excluded. The data gathered followed a standard form including the following variables: age, gender, admission weight, admission date, hospitalization time, antifungal used in the treatment, dose, time of treatment, date of collection and date of blood culture result, type of microbiological agent, 
antifungal sensitivity test, time of starting empiric therapy, adequacy based on antifungal susceptibility testing, prescribed dose as recommended by the institutional protocol for invasive fungal infections, and in-hospital outcome.

Patients' primary comorbidities were identified, as well as the presence of risk factors for candidemia (intensive care unit admission, mechanical ventilation, hemodialysis, blood transfusion, neutropenia (total neutrophil count $<500 / \mathrm{mm}^{3}$ ), use of vasoactive drugs, antimicrobial agents, corticosteroids, chemotherapy drugs, parenteral nutrition, surgical procedure, presence of central venous catheter). Breakthrough cases were also identified, defined as a positive blood culture during antifungal therapy of at least three days duration. These breakthrough cases were also included in our analysis [26, 27].

A candidemia episode was defined as the isolation of Candida in blood culture. For all patients, the first episode of candidemia was the episode analyzed. Polymicrobial blood cultures, that are where there was growth of other microorganisms in addition to Candida species, were excluded from the analysis.

Empirical therapy was defined as the initiation of an antifungal drug without knowledge of the infectioncausing microorganism species, and targeted therapy was defined as treatment with an antifungal drug after the culture result was reported.

Empirical therapy was considered inadequate when no intravenous antifungal agent was administered within $24 \mathrm{~h}$ of blood culture $[11,28]$. After obtaining the blood culture result identifying the microorganism and the antifungal susceptibility testing, therapy was considered inadequate if the isolated organism was resistant to the antifungal agent in use (for example, fluconazole for Candida krusei), if the antifungal agent was used in inadequate doses (e.g. fluconazole doses of less than $400 \mathrm{mg}$ per day) or if no antifungal agent was initiated $[10,11,29]$.

The hospital has an institutional protocol to guide the clinical staff based on international antifungal guidelines. Doses recommended by the institutional protocol were as follows: liposomal amphotericin B 3 to $5 \mathrm{mg} / \mathrm{kg} /$ day, fluconazole 400 to $800 \mathrm{mg} /$ day orally or intravenously, voriconazole $6 \mathrm{mg} / \mathrm{kg}$ IV every $12 \mathrm{~h}$ on day 1 followed by $4 \mathrm{mg} / \mathrm{kg} /$ day intravenously or orally, caspofungin loading dose of $70 \mathrm{mg}$ followed by $50 \mathrm{mg} /$ day thereafter, anidulafungin $200 \mathrm{mg}$ loading dose followed by $100 \mathrm{mg} /$ day thereafter, and micafungin $100 \mathrm{mg} /$ day $[16,30]$.

\section{Microbiological analysis}

All positive samples were identified using BACTEC FX equipment (BD Diagnostic Systems, Franklin Lakes, NJ, USA), an automated method used to detect microorganisms in blood cultures. After yeast were identified by gram stain, the chromogenic agar ChromID CPS (bioMérieux) was used, allowing identification of yeasts by the colors produced in the medium. Until 2013, species identification was carried out by VITEK 2 (bioMérieux) and from then on, to the present date, mass spectrometry VITEK MS (bioMérieux, Marcy l'Etoile, France) and Maldi-TOF (Bruker Microflex LT Biotyper 3.1) was used.

The antifungal susceptibility test profile was carried out for all blood culture samples positive for Candida, using the broth microdilution method according to CLSI document M27-A3, using current CLSI MIC interpretative criteria as M27-S4 [31, 32]. Candida parapsilosis ATCC22019 and Candida krusei ATCC6258 were used as quality controls. Commonly tested drugs were amphotericin B, fluconazole, voriconazole, anidulafungin (or caspofungin, depending on laboratory availability). Micafungin susceptibility testing was not performed because the laboratory did not have the specific test supplies for this drug during the study period.

\section{Statistical analysis}

In order to compare the DDD and DOT measurements, and to assess possible trends over time, Spearman correlation coefficients were used. In the comparison between hospitalization units in terms of antifungal consumption metrics, Kruskal-Wallis hypothesis tests were used, with post-tests corrected by the Steel [33] method, considering the medical and surgical clinic as a reference category.

Anidulafungin was introduced in the hospital formulary in 2010, micafungin in 2011 and posaconazole in 2016. These periods without consumption of these drugs were considered missing in the analysis. For the analysis of variations over time and for comparisons between units, if a unit had not consumed a certain antifungal in any valid year, the DDD and DOT values were considered zero. For correlations between DDD and DOT, zero values were not considered, but only the values or the years in which there was some antifungal consumption.

In order to investigate the in-hospital mortality associated factors, simple and multiple logistic regression models were fitted. For the multiple model, a stepwise variable selection process was conducted in both directions, considering information on the patient's profile, clinical history, antifungal treatment and hospitalization. The criterion for selection of variables was Akaike information (AIC). Model results are represented by $p$ values, odds ratios (OR) and their $95 \%$ confidence intervals $(95 \% \mathrm{CI})$. The analyses were performed using R 3.1.3 (R Core Team, 2015). The significance level adopted was $5 \%$.

\section{Results}

We found high correlation between the DDD and the study period indicating that there was a significant increase in the 
consumption of amphotericin B (rho ${ }_{\mathrm{DDD}}=0.87$ ) over time, particularly the liposomal formulation $\left(\mathrm{rho}_{\mathrm{DDD}}=0.83\right)$, micafungin $\left(\right.$ rho $_{\mathrm{DDD}}=0.89$ ), and intravenous voriconazole $\left(\right.$ rho $\left._{\text {DDD }}=0.80\right)$. There was a significant reduction in the consumption of caspofungin $\left(\mathrm{rho}_{\mathrm{DDD}}=-0.80\right.$ ) and intravenous fluconazole $\left(\mathrm{rho}_{\mathrm{DDD}}=-0.88\right)$ over time. The temporal DOT trends followed along the same DDD lines, but with some differences in intensity and significance. Figure 1 shows the antifungal consumption using DOT throughout the study period from 2008 to 2016 .

In investigating the correlation between DDD and DOT, the values of the correlation coefficients indicated positive correlations: the higher the DDD, the higher the DOT, but the correlation was not significant for all drugs.

Table 1 shows there was a significant difference between the utilization of all antifungal agents studied in the different hospitalization units, both according to DDD and DOT measurements. The consumption of posaconazole was not analyzed because it had only one measure in 2016.

The most commonly used antifungal drug was fluconazole, followed by liposomal amphotericin B, and the units with the highest consumption of these drugs were the Bone Marrow Transplantation, Oncology, Adult Intensive Care, and Solid Organ Transplantation Units (Table 1).

In the period between 2008 and 2016 there were 11,273 hospitalizations with at least one antifungal prescribed to 6779 patients who had one to 25 admissions within the study period. In $64.9 \%$ of admissions with antifungal prescriptions, a blood culture test was requested, which was positive for Candida in $3.2 \%$ of cases.

One hundred fifteen patients developed candidemia between 2012 and 2016. The majority (61.7\%) were men and the mean age was 68 years (SD: 17.9). Half of the patients died (51.3\%) and mean length of stay of 36 days. Table 2 also shows the risk factors associated with candidemia present during hospitalization and the clinical outcomes.

In $13.9 \%(16 / 115)$ of patients with candidemia antifungals were not used. Eight of these cases died before blood cultures were obtained, four were discharged from the hospital before the results of blood culture returned (two of which were readmitted), and for the others we did not find reasons in the patient's records for not treating the candidemia.

The duration of treatment with antifungals ranged from less than a full day to 149 days with a median of 14 days. The most commonly used antifungal agents were fluconazole $(42.6 \%, 49 / 115)$, followed by micafungin $(35.7 \%, 41 / 115)$ and caspofungin $(24.3 \%, 28 / 115)$. The most heavily used class of antifungals was echinocandins $(62.6 \%, 72 / 115)$. Each patient may have consumed more than one type of antifungal; there were cases of antifungal substitution during the same treatment, and in one patient there was a combination of two antifungals administered at the same time without justification in the medical record. As a result, $28.7 \%$ (33/115) of the patients received echinocandins only, 19. $1 \%(22 / 115)$ azoles only and 4.3\% (5/115) liposomal amphotericin B only. The Candida species most prevalent in blood cultures was C. albicans (36.5\%, 42/115), followed by $C$. parapsilosis $(22.6 \%, 26 / 115), C$. glabrata (21.7\%, 25/115), C. tropicalis $(11.3 \%, 13 / 115)$, C. krusei (4.3\%, 5/115), C. guilliermondii $(1.7 \% / 115)$. Two only species accounted for one case each.

Empiric therapy with antifungal drugs after blood cultures were drawn was started within $24 \mathrm{~h}$ in only $44.3 \%$ of the cases (Table 3 ). In $81.7 \%$ of cases (94/115), the empiric treatment was determined to be adequate after antifungal susceptibility tests were reviewed. The antifungal agent was changed in $36.5 \%$ of the cases. The first choice, in general, was fluconazole $(44.4 \%, 44 / 99)$, caspofungin $(22.2 \%, 22 / 99)$ or micafungin $(22.2 \%, 22 / 99)$, and the most commonly used antifungal after switching was

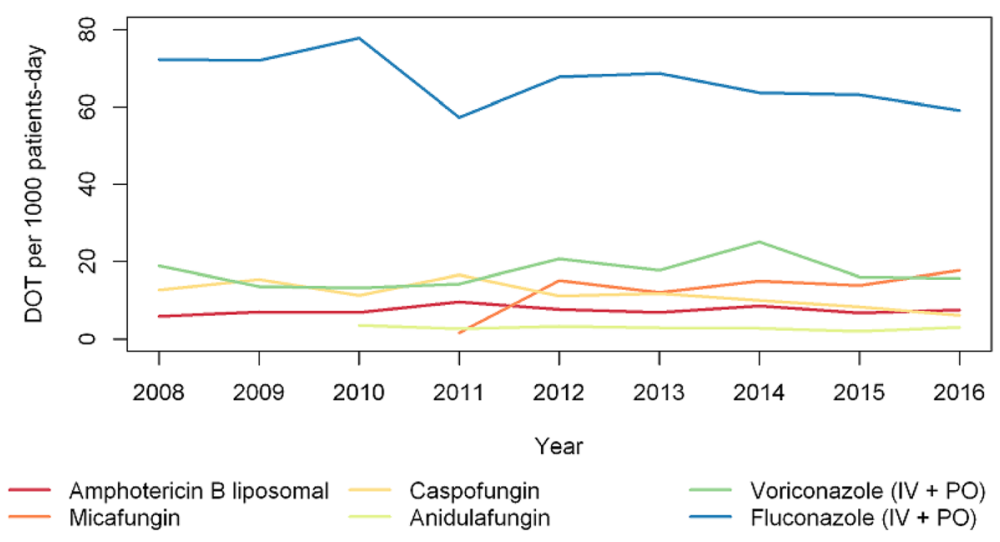

Fig. 1 Days of Therapy (DOT) per 1000 patient-days from 2008 to 2016. IV: intravenous; PO: oral 
Table 1 Median antifungal consumption in hospital units between 2008 and 2016

\begin{tabular}{|c|c|c|c|c|c|c|c|c|}
\hline Drug\Unit & MSC & Onco & Ped & AICU & BMT & TX & $\mathrm{NICU}$ & $P$ Value* \\
\hline \multicolumn{9}{|l|}{ DDD per 1000 patient-days } \\
\hline Amphotericin B (lipos + deox) & 14.2 & 190.9 & & 239.4 & 359.0 & 79.2 & & $<0.001$ \\
\hline$P$ Value & & 0.002 & & 0.002 & 0.002 & 0.045 & & \\
\hline Amphotericin B liposomal & 13.8 & 190.9 & & 237.5 & 351.3 & 78.9 & & $<0.001$ \\
\hline$P$ Value & & 0.002 & & 0.002 & 0.002 & 0.110 & & \\
\hline Amphotericin B deoxycholate & 0.4 & 0.0 & & 3.2 & 0.0 & 7.9 & & 0.012 \\
\hline$P$ Value & & 0.904 & & 0.110 & 0.940 & 0.135 & & \\
\hline Anidulafugin (2010-2016) & 1.4 & 11.6 & & 20.0 & 6.2 & 0.0 & & 0.002 \\
\hline$P$ Value & & 0.009 & & 0.009 & 0.995 & 0.568 & & \\
\hline Caspofungin & 7.2 & 28.1 & & 73.3 & 46.7 & 2.0 & & $<0.001$ \\
\hline$P$ Value & & 0.007 & & 0.002 & 0.057 & 0.110 & & \\
\hline Fluconazole (IV + PO) & 35.7 & 257.9 & & 160.7 & 625.6 & 159.2 & & $<0.001$ \\
\hline$P$ Value & & 0.002 & & 0.002 & 0.002 & 0.002 & & \\
\hline Fluconazole L IV & 25.3 & 163.3 & & 150.7 & 382.3 & 52.6 & & $<0.001$ \\
\hline$P$ Value & & 0.002 & & 0.002 & 0.002 & 0.002 & & \\
\hline Fluconazole PO & 11.4 & 124.9 & & 14.3 & 134.2 & 102.8 & & $<0.001$ \\
\hline$P$ Value & & 0.002 & & 0.035 & 0.002 & 0.002 & & \\
\hline Itraconazole & 0.7 & 2.7 & & 0.6 & 0.00 & 14.7 & & 0.001 \\
\hline$P$ Value & & 0.625 & & 0.690 & 0.006 & 0.324 & & \\
\hline Micafungina (2011-2016) & 3.7 & 41.8 & & 71.7 & 153.6 & 21.2 & & 0.001 \\
\hline$P$ Value & & 0.020 & & 0.077 & 0.020 & 0.224 & & \\
\hline Posaconazole (2016) & 0.0 & 44.4 & & 6.9 & 87.3 & 0.0 & & - \\
\hline Voriconazole (IV + PO) & 3.1 & 82.6 & & 22.2 & 174.4 & 7.1 & & $<0.001$ \\
\hline$P$ Value & & 0.002 & & 0.002 & 0.002 & 0.495 & & \\
\hline Voriconazole IV & 1.7 & 44.4 & & 16.0 & 83.0 & 3.7 & & $<0.001$ \\
\hline$P$ Value & & 0.002 & & 0.002 & 0.002 & 0.993 & & \\
\hline Voriconazole PO & 1.4 & 42.4 & & 5.4 & 80.6 & 3.4 & & $<0.001$ \\
\hline$P$ Value & & 0.002 & & 0.007 & 0.002 & 0.324 & & \\
\hline \multicolumn{9}{|l|}{ DOT per 1000 patient-days } \\
\hline Amphotericin B (lipos + deox) & 3.2 & 29.8 & 20.2 & 38.9 & 85.2 & 16.0 & 2.6 & $<0.001$ \\
\hline$P$ Value & & 0.002 & 0.016 & 0.002 & 0.002 & 0.016 & 0.943 & \\
\hline Amphotericin B liposomal & 2.5 & 29.8 & 19.7 & 36.0 & 82.8 & 10.8 & 0.5 & $<0.001$ \\
\hline$P$ Value & & 0.002 & 0.004 & 0.002 & 0.002 & 0.134 & 0.069 & \\
\hline Amphotericin B deoxycholate & 0.4 & 0.0 & 0.0 & 1.6 & 0.0 & 5.2 & 1.1 & $<0.001$ \\
\hline$P$ Value & & 0.745 & 0.100 & 0.045 & 0.966 & 0.028 & 0.434 & \\
\hline Anidulafugin (2010-2016) & 1.3 & 11.2 & 0.0 & 18.4 & 5.4 & 0.0 & 0.0 & $<0.001$ \\
\hline$P$ Value & & 0.009 & 0.377 & 0.009 & 0.995 & 0.568 & 0.005 & \\
\hline Caspofungin & 7.0 & 27.0 & 15.0 & 65.4 & 45.9 & 1.8 & 0.0 & $<0.001$ \\
\hline$P$ Value & & 0.007 & 0.057 & 0.002 & 0.089 & 0.057 & 0.001 & \\
\hline Fluconazole (IV + PO) & 42.9 & 237.0 & 41.5 & 147.7 & 420.1 & 174.4 & 1.1 & $<0.001$ \\
\hline$P$ Value & & 0.002 & 0.984 & 0.002 & 0.002 & 0.002 & 0.002 & \\
\hline Fluconazole L IV & 23.4 & 102.0 & 29.8 & 126.7 & 259.4 & 51.1 & 1.1 & $<0.001$ \\
\hline$P$ Value & & 0.002 & 0.690 & 0.002 & 0.002 & 0.002 & 0.002 & \\
\hline
\end{tabular}


Table 1 Median antifungal consumption in hospital units between 2008 and 2016 (Continued)

\begin{tabular}{|c|c|c|c|c|c|c|c|c|}
\hline Drug\Unit & MSC & Onco & Ped & $\mathrm{AlCU}$ & BMT & TX & $\mathrm{NICU}$ & $P$ Value* \\
\hline Fluconazole PO & 20.1 & 130.4 & 13.2 & 23.6 & 160.6 & 119.7 & 0.0 & $<0.001$ \\
\hline$P$ Value & & 0.002 & 0.057 & 0.434 & 0.002 & 0.002 & 0.001 & \\
\hline Itraconazole & 0.8 & 1.2 & 1.0 & 0.8 & 0.0 & 11.8 & 0.0 & $<0.001$ \\
\hline$P$ Value & & 0.753 & 1.000 & 0.812 & 0.008 & 0.324 & 0.001 & \\
\hline Micafungina (2011-2016) & 3.9 & 45.3 & 8.5 & 73.5 & 225.5 & 21.8 & 0.0 & $<0.001$ \\
\hline$P$ Value & & 0.020 & 0.624 & 0.077 & 0.020 & 0.224 & 0.024 & \\
\hline Posaconazole (2016) & 0.0 & 3.1 & 0.0 & 0.5 & 6.2 & 0.0 & 0.0 & - \\
\hline Voriconazole (IV + PO) & 5.4 & 130.1 & 35.0 & 25.4 & 322.3 & 10.1 & 0.00 & $<0.001$ \\
\hline$P$ Value & & 0.002 & 0.002 & 0.002 & 0.002 & 0.559 & 0.001 & \\
\hline Voriconazole IV & 2.6 & 57.2 & 14.0 & 20.1 & 142.7 & 4.7 & 0.00 & $<0.001$ \\
\hline$P$ Value & & 0.002 & 0.002 & 0.002 & 0.002 & 0.984 & 0.001 & \\
\hline Voriconazole PO & 2.2 & 73.7 & 17.7 & 7.1 & 147.7 & 5.6 & 0.00 & $<0.001$ \\
\hline$P$ Value & & 0.002 & 0.028 & 0.002 & 0.002 & 0.276 & 0.001 & \\
\hline
\end{tabular}

DDD Defined Daily Dose, DOT Days of Therapy, IV Intravenous, PO Oral, MSW Medical and Surgical Wards, Onco Oncology, Ped Pediatrics, AICU Adult Intensive Care Unit, BTM Bone marrow transplant, SOT Solid organ transplant, NICU Neonatal Intensive Care Unit *P values for Kruskal-Wallis tests to test whether in at least one of the units the consumption was different from the others. Other $p$-values for post-tests with Steel correction that compare each unit with the Medical and Surgical Wards

micafungin (17.4\%, 20/115), followed by liposomal amphotericin B (13\%, 15/115). Table 3 also shows cases where the dose was adequate according to institutional protocol.

As for the clinical history, 59.1\% (68/115) of patients suffered from cardiac disease, $27.8 \%(32 / 115)$ from diabetes mellitus, 23.5\% (27/115) from nephropathy, $22.6 \%$ (26/115) from cancer, and $19.1 \%(22 / 115)$ were bone marrow and solid organ transplant patients.

In Table 4 are the variables that were evaluated by simple logistic regression analysis. In Table 5, after the construction of multiple logistic regression analysis, the factors that remained significant and associated with death risk were: age, length of stay in the ICU, use of vasoactive drugs, use of chemotherapy, hemodialysis and positive blood culture while on antifungal therapy (breakthrough candidemia).

\section{Discussion}

Analyzing the data, it was possible to identify a wide use of antifungal drugs, which may be related to the characteristics of the hospital, because it is large tertiary care facility This study is similar to a study carried out in Spain, where antifungal consumption was higher in large university hospitals [34].

In our study we observed a significant increase over time in the consumption of liposomal amphotericin $\mathrm{B}$, micafungin and voriconazole, and a significant reduction in the consumption of caspofungin and fluconazole (Fig. 1). The increased use of liposomal amphotericin B and voriconazole is probably related to the empirical and prophylactic treatments used in the onco-hematology unit, and the increase of micafungin is due to its lower cost. We also suspect that the reduction of fluconazole usage may be related to the predominance of $C$. non-albicans species in our institution, and the reduction in the use of caspofungin due to the higher cost of this drug and the standardization of use of micafungin, which has lower cost and equivalent effectiveness. We believe that introducing an agent of the same class at a lower cost into the hospital formulary has caused this change in the pattern of echinocandin prescriptions in our institution over the years. Since studies do not show significant differences in efficacy among these drugs, cost may become a differential point in decision making over which drug to choose. Our findings were different from the Spanish study, where there was an increase in fluconazole consumption, followed by a smaller scale increase in voriconazole, and a reduction in itraconazole consumption [34]. In the same study, the consumption of echinocandins was more apparent in the ICU; we also observed in our study that the adult ICU had the highest consumption of caspofungin and anidulafungin (Table 1). In another study conducted in ICUs, a decrease in the use of fluconazole and an increase in echinocandin consumption also became evident over time [35]. In a Brazilian university hospital, the highest consumption was of the azole class, first fluconazole, followed by voriconazole and the third most consumed was amphotericin B deoxycholate, a fact that can be explained by the limitations in resources, since it is a public hospital, and this deoxycholate formulation is cheaper, with greater control over the use of liposomal amphotericin $\mathrm{B}$ and echinocandins, requiring 
Table 2 Hospital admission data and risk factors for patients with candidemia $(N=115)$

\begin{tabular}{|c|c|}
\hline Variables & Descriptions \\
\hline \multicolumn{2}{|l|}{ Sex $n(\%)$} \\
\hline Female & $44(38.3)$ \\
\hline Male & $71(61.7)$ \\
\hline \multicolumn{2}{|l|}{ Age (years) } \\
\hline Mean (SD) & $68(17.9)$ \\
\hline Median (1st quartile - 3rd quartile) & $70[56-84]$ \\
\hline Minimum maximum & $26-100$ \\
\hline \multicolumn{2}{|l|}{ Length of stay (days) } \\
\hline Mean (SD) & $69.8(138.4)$ \\
\hline Median (1st quartile - 3rd quartile) & $36[21-62.5]$ \\
\hline Minimum maximum & $1-1003$ \\
\hline Use of antimicrobial & $113(98.3)$ \\
\hline Use of chemotherapy drugs & $12(10.4)$ \\
\hline Use of corticosteroids & $95(82.6)$ \\
\hline Use of parenteral nutrition & $46(40.0)$ \\
\hline Presence of some type of central venous catheter & $86(74.8)$ \\
\hline Had any type of surgery & $79(68.7)$ \\
\hline Admission to the intensive care unit & $94(81.7)$ \\
\hline \multicolumn{2}{|l|}{ Length of ICU stay (days) $(n=94)$} \\
\hline Mean (SD) & $23.3(23.3)$ \\
\hline Median (1st quartile - 3rd quartile) & $15(6-32)$ \\
\hline Minimum maximum & $1-100$ \\
\hline Neutropenia & $6(5.2)$ \\
\hline Use of vasoactive drug & $86(74.8)$ \\
\hline Use of mechanical ventilation & $82(71.3)$ \\
\hline Hemodialysis & $38(33.0)$ \\
\hline Had hemotherapy & $27(23.5)$ \\
\hline Positive blood culture even when using antifungal therapy & $35(30.4)$ \\
\hline \multicolumn{2}{|l|}{ Clinical outcome } \\
\hline Discharge from hospital & $56(48.7)$ \\
\hline In-hospital mortality & $59(51.3)$ \\
\hline
\end{tabular}

Category variables presented by absolute and relative frequencies. $S D$ standard deviation

authorization prior to the prescription of these highcost drugs [36].

Two other German studies used different metrics, one Recommended Daily Dose (RDD) and the other Prescribed Daily Dose (PDD), and both showed a higher consumption of antifungal drugs in the ICU and Hematology/Oncology service [37, 38]. Our study shows that these units also have a high consumption of antifungal agents (Table 1), since antifungal prophylaxis is included in the treatment profile of these patients and the use of empiric therapy is quite common $[38,39]$.
Table 3 Empiric therapy in patients with candidemia $(N=115)$

\begin{tabular}{ll}
\hline Variables & $n(\%)$ \\
\hline Time to start empiric therapy with antifungal after blood culture \\
obtained (hours) & $51(44.3)$ \\
24 & $20(17.4)$ \\
48 & $16(13.9)$ \\
72 & $7(6.1)$ \\
96 & $5(4.3)$ \\
120 & $16(13.9)$ \\
No use of antifungal drug & $21(18.3)$ \\
Appropriate empirical antifungal treatment according to antifungal \\
susceptibility test result \\
No & $94(81.7)$ \\
Yes & $28 / 28(100.0)$ \\
Adequate dose according to institutional protocol & \\
Caspofungin & $31 / 41(75.6)$ \\
Micafungin & $5 / 8(62.5)$ \\
Anidulafungin & $12 / 49(24.5)$ \\
Fluconazole & $20 / 21(95.2)$ \\
Amphotericin B liposomal & $4 / 5(80.0)$ \\
Voriconazole &
\end{tabular}

Category variables presented by absolute and relative frequencies

a number of cases observed/number of cases using antifungal drug

When comparing our work with an earlier study performed at the same institution [1], where the DDD of antifungal drugs was calculated from 1997 to 2007, we can observe that, in general, there was an increase in the consumption of these drugs, but there was also an increase in the therapeutic arsenal available in our formulary. In 2010, anidulafungin was added, micafungin was added in 2011, and posaconazole in 2016. Fluconazole consumption remained stable, mean use 61.5 (range from 32.7 to 101.2) DDD/1000 patient-days in the first study, and mean use 62.14 (range from 50.11 to 70.66) $\mathrm{DDD} / 1000$ patient-days in the second study. Observation demonstrated that the use of amphotericin B deoxycholate continued to be reduced in our study, as in the previous one, due to the higher risk of nephrotoxicity associated with this formulation, which led to an increase in the prescription of liposomal amphotericin $\mathrm{B}$ and echinocandins.

For most drugs there was a positive correlation between DDD and DOT values, but not all of them were strong and significant. A different pattern was observed for amphotericins, which we believe to be due to the fact that when the dose administered exceeds the Individualized Daily Dose (IDD), the DDD significantly exceeds the DOT. For drugs such as caspofungin and micafungin, there is usually no significant difference between DDD and DOT per 1000 patient-days, as the daily administered dose is close to the IDD equivalent [23]. 
Table 4 Data for hospitalization and risk factors, by type of discharge $(N=115)$

\begin{tabular}{|c|c|c|c|c|}
\hline & Discharge $(N=56)$ & $\begin{array}{l}\text { In-hospital } \\
\text { mortality }(N=59)\end{array}$ & OR $(95 \% \mathrm{Cl})$ & $P$ value \\
\hline \multicolumn{5}{|l|}{ Gender } \\
\hline Female & $23(41.1)$ & $21(35.6)$ & 1.00 & \\
\hline Male & $33(58.9)$ & $38(64.4)$ & $1.26(0.59 ; 2.69)$ & 0.546 \\
\hline Age (years) & & & $1.03(1.01 ; 1.05)$ & 0.015 \\
\hline Mean (SD) & $63.80(19.33)$ & $72.03(15.53)$ & & \\
\hline Median [1st Q - 3rd Q] & $65.00[50-81.50]$ & $76.00[60-85]$ & & \\
\hline Length of stay (days) & & & $1.00(1.00 ; 1.00)$ & 0.952 \\
\hline Mean (SD) & $69.02(140.67)$ & $70.58(137.32)$ & & \\
\hline Median [1st Q - 3rd Q] & $32[20.25-64.25]$ & $36[23.50-56]$ & & \\
\hline \multicolumn{5}{|c|}{ Time to start empiric therapy with antifungal after blood culture } \\
\hline Within $24 \mathrm{~h}$ & $23(41.1)$ & $28(47.5)$ & 1.00 & \\
\hline More than $24 \mathrm{~h}$ & $27(48.2)$ & $21(35.6)$ & $0.64(0.29 ; 1.41)$ & 0.268 \\
\hline No use of antifungal drug & $6(10.7)$ & $10(16.9)$ & $1.37(0.44 ; 4.56)$ & 0.593 \\
\hline \multicolumn{5}{|l|}{ Used antimicrobial } \\
\hline No & $1(1.8)$ & $1(1.7)$ & 1.00 & \\
\hline Yes & $55(98.2)$ & $58(98.3)$ & - 11-22-2016 17:19:0 s2 & 0.970 \\
\hline \multicolumn{5}{|l|}{ Used chemotherapy } \\
\hline No & $52(92.9)$ & $51(86.4)$ & 1.00 & \\
\hline Yes & $4(7.1)$ & $8(13.6)$ & $2.04(0.60 ; 8.03)$ & 0.268 \\
\hline \multicolumn{5}{|l|}{ Used corticosteroid } \\
\hline No & $15(26.8)$ & $5(8.5)$ & 1.00 & \\
\hline Yes & $41(73.2)$ & $54(91.5)$ & $3.95(1.40 ; 12.96)$ & 0.014 \\
\hline \multicolumn{5}{|l|}{ Used parenteral nutrition } \\
\hline No & $37(66.1)$ & $32(54.2)$ & 1.00 & \\
\hline Yes & 19 (33.9) & $27(45.8)$ & $1.64(0.78 ; 3.53)$ & 0.197 \\
\hline \multicolumn{5}{|l|}{ Used some type of central venous catheter } \\
\hline No & $22(39.3)$ & $7(11.9)$ & 1.00 & \\
\hline Yes & $34(60.7)$ & $52(88.1)$ & $4.81(1.93 ; 13.30)$ & 0.001 \\
\hline \multicolumn{5}{|l|}{ Had any type of surgery } \\
\hline No & $20(35.7)$ & $16(27.1)$ & 1.00 & \\
\hline Yes & $36(64.3)$ & $43(72.9)$ & $1.49(0.68 ; 3.33)$ & 0.322 \\
\hline \multicolumn{5}{|l|}{ Stayed at the intensive care unit } \\
\hline No & $17(30.4)$ & $4(6.8)$ & 1.00 & \\
\hline Yes & $39(69.6)$ & $55(93.2)$ & $5.99(2.04 ; 22.07)$ & 0.003 \\
\hline Length of stay in the intensive care unit (days) $(n=94)$ & & & $1.04(1.02 ; 1.06)$ & 0.001 \\
\hline Mean (SD) & $16.03(16.86)$ & $28.42(25.86)$ & & \\
\hline Median [1st Q - 3rd Q] & $10.00(5.00-17.50)$ & $23.00(7.50-36.00)$ & & \\
\hline \multicolumn{5}{|l|}{ Neutropenia } \\
\hline No & $54(96.4)$ & $55(93.2)$ & 1.00 & \\
\hline Yes & $2(3.6)$ & $4(6.8)$ & $1.96(0.37 ; 14.59)$ & 0.447 \\
\hline \multicolumn{5}{|l|}{ Used vasoactive drug } \\
\hline No & $23(41.1)$ & $6(10.2)$ & 1.00 & \\
\hline Yes & $33(58.9)$ & $53(89.8)$ & $6.16(2.39 ; 18.12)$ & $<0.001$ \\
\hline
\end{tabular}


Table 4 Data for hospitalization and risk factors, by type of discharge $(N=115)$ (Continued)

\begin{tabular}{|c|c|c|c|c|}
\hline & Discharge $(N=56)$ & $\begin{array}{l}\text { In-hospital } \\
\text { mortality }(N=59)\end{array}$ & OR $(95 \% \mathrm{Cl})$ & $P$ value \\
\hline \multicolumn{5}{|c|}{ Used mechanical ventilation } \\
\hline No & $26(46.4)$ & $7(11.9)$ & 1.00 & \\
\hline Yes & $30(53.6)$ & $52(88.1)$ & $6.44(2.61 ; 17.74)$ & $<0.001$ \\
\hline \multicolumn{5}{|c|}{ Hemodialysis } \\
\hline No & $45(80.4)$ & $32(54.2)$ & 1.00 & \\
\hline Yes & $11(19.6)$ & $27(45.8)$ & $3.45(1.53 ; 8.21)$ & 0.004 \\
\hline \multicolumn{5}{|c|}{ Blood transfusion } \\
\hline No & $48(85.7)$ & $40(67.8)$ & 1.00 & \\
\hline Yes & $8(14.3)$ & $19(32.2)$ & $2.85(1.16 ; 7.55)$ & 0.027 \\
\hline \multicolumn{5}{|c|}{ Positive blood culture even when using antifungal therapy } \\
\hline No & $44(78.6)$ & $36(61.0)$ & 1.00 & \\
\hline Yes & $12(21.4)$ & $23(39.0)$ & $2.34(1.04 ; 5.48)$ & 0.043 \\
\hline
\end{tabular}

Category variables presented by absolute and relative frequencies. $Q$ quartile $S D$ standard deviation, OR odds ratio obtained by simple logistic regression model (values, 1.00 for the reference categories) and, in parentheses, the $95 \%$ Confidence Interval $(95 \% \mathrm{Cl})$

The ideal metric to measure drug consumption is still not well defined in the literature. The two metrics used in this study have both advantages and disadvantages, and most studies involve the consumption of antibacterial drugs. A primary concern with this class of drugs is bacterial resistance, which is increasing while the development of new agents is slow. The emergence of antifungal resistance should prompt the monitoring of utilization of these drugs.

DDD allows the comparison of utilization in different hospitals, regions and countries. It can be calculated even when a computerized pharmacy data system is not available, although electronic systems aids in optimizing the calculation time by the pharmacist in both metrics $[23,25]$. Disadvantages of DDD are its inaccuracy in drugs that require dose adjustment for renal function or weight-based drugs. IDD values may change with the approval of new doses for existing drugs, which can create

Table 5 Factors associated with death risk in patients with candidemia $(\mathrm{N}=115)$

\begin{tabular}{lll}
\hline Factors & OR $(95 \% \mathrm{Cl})$ & $P$ value \\
\hline Age (years) & $1.04(1.01 ; 1.08)$ & 0.005 \\
Treatment with antifungal (days) & $0.95(0.91 ; 0.99)$ & 0.022 \\
Time in ICU (days) & $1.03(1.01 ; 1.07)$ & 0.020 \\
Use of vasoactive drugs (Yes) & $3.90(1.18 ; 14.43)$ & 0.031 \\
Use of chemotherapy (Yes) & $6.88(1.37 ; 40.17)$ & 0.023 \\
Hemodialysis (Yes) & $3.19(1.11 ; 9.86)$ & 0.035 \\
Positive blood culture even & $4.20(1.25 ; 15.86)$ & 0.026 \\
when using antifungal drug (Yes) & & \\
\hline
\end{tabular}

In parentheses, the $95 \%$ Confidence Interval (95\% Cl). OR odds ratio obtained by multiple logistic regression model confusion when comparisons of use are made over time $[23-25,40]$. The advantages of the DOT metric are greater accuracy than DDD, it can be used to measure utilization in children (weight-based regimens), is not influenced in case of changes in recommended doses of IDD, is not influenced by discrepancies between IDD and preferred daily doses or usual daily doses, which may vary between hospitals $[23,38]$. The disadvantages of DOT are: calculation is more difficult without computerized systems and there is a lack of accuracy in populations with renal failure, although the values obtained with DOT calculation are more accurate than when calculating DDD in this population [23, 25].

The literature recognizes the limitations regarding the use of DDD in pediatric patients, so some authors believe that DOT is more appropriate for this population, particularly for low weight patients [17, 23, 40].

Regarding microbiological characteristics, the Brazilian study published in 2016 [3] also showed an increase in the occurrence Candida non-albicans, corresponding to $65.7 \%$ of the cases, although in isolation C. albicans still remains as the predominant species at $34.3 \%$, followed by $C$. parapsilosis (24.1\%), C. tropicalis (15.3\%) and C. glabrata (10.2\%). These results are similar to those of our study, where $C$. albicans the most isolated species corresponded $36.5 \%$ of the cases, followed by C. parapsilosis (22.6\%), C. glabrata (21.7\%) and C. tropicalis (11.3\%), differing only in the rank order and percentages for C. tropicalis and C. glabrata. In the prior study carried out in our institution from 1997 to 2007, C. albicans was the most isolated species in blood cultures (44\%), followed by C. parapsilosis (22\%), C. tropicalis (15\%) and C. glabrata (9\%) [1]. In view of these data, over the years we can see the increase of $C$. non-albicans species in our institution as well, as reported in other studies [5, 41, 42]. 
We did not analyze the change in antifungal susceptibilities over the years in this study, but we could make a comparison with a prior study carried out in this institution from 1997 to 2007 whose susceptibility testing data are from mid-2002 to 2007 [1]. This showed that the resistance profile remained proportionally the same, with $100 \%$ of strains susceptible to amphotericin B and $91.4 \%$ ( $n=96 / 105)$ of the strains susceptible to fluconazole, compared to $82.3 \%$ in the prior study $(n=42 / 51)$. Table 6 shows the susceptibility tests of Candida species isolates found in the first study conducted at our institution, data from mid-2002 to 2007 [1] and the findings in our study from 2012 to 2016. Among the resistant and intermediate species (susceptible dose-dependent) are $C$. krusei and C. glabrata, respectively. Until 2007, we only had caspofungin on formulary at our institution, and until then no susceptibility tests were found with intermediate results for this drug. However, in the current study, we have already seen the appearance of intermediate susceptibility to echinocandins, although it is a small percentage of strains, we can consider this as a warning sign to prompt increased monitoring of the use of these class of drugs, since the use of antifungal drugs affects the susceptibility of Candida species [43] and the selection of resistant strains may be responsible for failures in treatment [44].

In a study carried out in Costa Rica [43], 19\% of adult patients with candidemia did not receive antifungal drugs and these patients had a significantly higher risk of death within the next 30 days when compared to patients receiving antifungal drugs (OR: $2.9,95 \%$ CI 1.8 to $4.6, p<0.001)$. In our study, $13.9 \%$ of patients who had candidemia did not receive antifungal drugs; however, treatment with antifungals was associated with reduced mortality in multivariate analysis. In the Costa Rican study [45], 82\% of candidemic patients started antifungal therapy more than $24 \mathrm{~h}$ after the blood culture was obtained. In our study the proportion was much smaller (41.7\%) and no significant association was found between the timing of empiric therapy and mortality. In a European study, the proportion of patients receiving adequate antifungal therapy in the first $24 \mathrm{~h}$ after blood culture was high, and the delay in initiating antifungal therapy was not a risk factor for death [46].

In another study, which involved patients in septic shock, no differences were found in mortality regarding the timing of the beginning of antifungal therapy, but they identified a higher mortality in the group of patients where antifungal therapy was not adequate $(\mathrm{p}<0$. 001) [12]. In our study, for most patients antifungal doses were in accordance with the institutional protocol but to our surprise the fluconazole dose was only adequate in $24.5 \%$ of the cases, with the majority being prescribed in suboptimal doses for the treatment of
Table 6 Comparison of microbiological data susceptibility tests between our study and Camargo et al. study [1]

\begin{tabular}{|c|c|}
\hline Variables & $n(\%)$ \\
\hline \multicolumn{2}{|l|}{2002 a $2007^{*}$} \\
\hline \multicolumn{2}{|c|}{ Candida albicans $(n=27)$} \\
\hline \multicolumn{2}{|c|}{ Amphotericin B $(n=27)$} \\
\hline Susceptible & $27(100.0)$ \\
\hline \multicolumn{2}{|c|}{ Fluconazole $(n=27)$} \\
\hline Susceptible & $27(100.0)$ \\
\hline \multicolumn{2}{|c|}{ Candida non-albicans $(n=51)$} \\
\hline \multicolumn{2}{|c|}{ Amphotericin B $(n=51)$} \\
\hline Susceptible & $51(100.0)$ \\
\hline \multicolumn{2}{|c|}{ Fluconazole $(n=51)$} \\
\hline Susceptible & $42(82.3)$ \\
\hline \multicolumn{2}{|l|}{2012 a $2016^{* *}$} \\
\hline \multicolumn{2}{|c|}{ Candida albicans ( $n=42)$} \\
\hline \multicolumn{2}{|c|}{ Amphotericin B $(n=40)$} \\
\hline Susceptible & $40(100.0)$ \\
\hline \multicolumn{2}{|c|}{ Fluconazole $(n=41)$} \\
\hline Susceptible & $41(100.0)$ \\
\hline \multicolumn{2}{|c|}{ Voriconazole $(n=41)$} \\
\hline Susceptible & $41(100.0)$ \\
\hline \multicolumn{2}{|c|}{ Caspofungin $(n=1)$} \\
\hline Susceptible & $1(100.0)$ \\
\hline \multicolumn{2}{|c|}{ Anidulafungin $(n=3)$} \\
\hline Susceptible & $3(100.0)$ \\
\hline \multicolumn{2}{|c|}{ Candida non-albicans $(n=73$ ) } \\
\hline \multicolumn{2}{|c|}{ Amphotericin B $(n=72)$} \\
\hline Susceptible & $72(100.0)$ \\
\hline \multicolumn{2}{|c|}{ Fluconazole $(n=64)$} \\
\hline Susceptible & $55(85.9)$ \\
\hline Resistant & $3(4.7)$ \\
\hline Intermediate & $6(9.4)$ \\
\hline \multicolumn{2}{|c|}{ Voriconazole $(n=64)$} \\
\hline Susceptible & $63(98.4)$ \\
\hline Intermediate & $1(1.6)$ \\
\hline \multicolumn{2}{|c|}{ Caspofungin $(n=10)$} \\
\hline Susceptible & $9(90.0)$ \\
\hline ntermediate & $1(10.0)$ \\
\hline \multicolumn{2}{|c|}{ Anidulafungin $(n=11)$} \\
\hline Susceptible & $8(72.7)$ \\
\hline Intermediate & $3(27.3)$ \\
\hline
\end{tabular}

Category variables presented by absolute and relative frequencies *Data from Camargo TZS, et al. study [1]. "Data from our current study

candidemia. The AmarCAND2 group study [47] also showed that the dose of fluconazole was not adequate in most patients. In view of these numbers, we can 
conclude that the pharmacist's performance in the verification of the medical prescription by checking the doses and frequencies of the medications is very important, since treatment with inadequate doses may lead to unfavorable clinical outcomes.

In another study there was no correlation between the reduction of in-hospital mortality and the time of initiation of the therapy or initial choice of agents; instead they observed that risk factors, such as age, admission to the ICU or diabetes mellitus, have a strong impact on outcomes [13]. On the other hand, another study identified that mortality was lower when fluconazole therapy was started on the same day the culture was obtained [11]. We observed that there are still differences in the literature regarding whether the appropriate therapy actually impacts the risk of death, but it is clear that there are other factors that strongly influence the mortality related to candidemia, such as the severity of illness and the presence of comorbidities [13, 48].

An American study identified through univariate analysis that the predictors of increased mortality included the APACHE II severity of illness score, intensive care unit admission, corticosteroid treatment, and the use of mechanical ventilation [11]. In our study, independent predictors of mortality age, ICU length of stay, use of vasoactive drugs, chemotherapy, hemodialysis, and breakthrough candidemia.

The Eurobact study [44] identified mechanical ventilation and ICU admission as independent risk factors for death. These variables were not independent predictors in our study, but the length of ICU stay was an independent predictor of mortality.

Our study has some limitations primarily that it is single center and retrospective. The retrospective nature of the study made it impossible to identify the reason for the choice of specific antifungal drugs as initial therapy or the reason for the drug exchange during antifungal therapy, and also it was not possible to identify the number of cases where the antifungal was used as prophylaxis. To reduce bias, we chose to include only microbiological data from blood cultures to evaluate adequacy of antifungal therapy, since for a positive culture is highly predictive of infection and requires intervention, which is not characteristic of other sites, such as the urinary or respiratory tracts, where positivity may be due to colonization, which makes results difficult to interpret.

\section{Conclusion}

The high utilization of antifungal drugs reinforces the need for ongoing monitoring, the implementation of stewardship interventions, and the key role of the pharmacist to increase rational use and thus improve patient safety.

\section{Abbreviations \\ AIC: Akaike information; AICU: Adult intensive care unit; APACHE II: Acute physiology and chronic health evaluation II; ATC: Anatomical therapeutic chemical; BMT: Bone marrow transplantation; Cl: Confidence intervals; DDD: Defined daily dose; IDD: Individualized daily dose; DOT: Days of therapy; ICU: Intensive care unit; MSW: Medical and surgical wards; NICU: Neonatal intensive care unit; ONCO: Oncology; OR: Odds ratios; PDD: Prescribed daily dose; PED: Pediatrics; RDD: Recommended daily dose; SOT: Solid organ transplantation; WHO: World Health Organization}

\section{Acknowledgements}

We are grateful to the Hospital Israelita Albert Einstein mainly for the Pharmacy Service and the Microbiology Laboratory for support this work.

\section{Availability of data and materials}

Confidentiality agreements prevent us from sharing the raw data generated during this study. The data is available upon request. The corresponding author should be contacted if someone wants to request the data.

\section{Authors' contributions}

TAM, PKOY, MDVM participated in the data collected. TAM, ARM, MBE, MSDJ, ACCNM participated in the data analysis. TAM, ARM, MBE, MSDJ participated in the design and coordination. TAM, ARM, MBE, MDVM, PKOY, ACCNM, MSDJ helped to draft the manuscript and to provide critical review to the manuscript. All authors read and approved the final manuscript.

\section{Ethics approval and consent to participate}

This study was approved by the Ethics Committee of the Instituto Israelita de Ensino e Pesquisa Albert Einstein. The requirements for informed consent were waived by our IRB in accordance of the Code of Federal Regulation and of the Privacy Rule.

\section{Competing interests}

The authors declare that they have no competing interests. This research received no specific grant from any funding agency in the public, commercial, or not-for-profit sectors.

\section{Publisher's Note}

Springer Nature remains neutral with regard to jurisdictional claims in published maps and institutional affiliations.

\section{Author details}

${ }^{1}$ Hospital Israelita Albert Einstein, São Paulo, Brazil. ${ }^{2}$ Division of Medical Practice, Hospital Israelita Albert Einstein, São Paulo, Brazil. ${ }^{3}$ Office of Clinical Quality, Safety and Performance Improvement, University of lowa Hospitals and Clinics, lowa City, IA, USA. ${ }^{4}$ Division of Infectious Diseases, Department of Internal Medicine, University of lowa Carver College of Medicine, lowa City, IA, USA. ${ }^{5}$ Clinical Laboratory, Hospital Israelita Albert Einstein, São Paulo, Brazil. ${ }^{6}$ Instituto Israelita de Ensino e Pesquisa Albert Einstein, Hospital Israelita Albert Einstein, São Paulo, Brazil.

Received: 16 November 2017 Accepted: 12 April 2018

Published online: 18 April 2018

\section{References}

1. Camargo TZS, Marra AR, Silva CV, Cardoso MFS, Martino MDV, Camargo LFA, Correa L. Secular trends of candidemia in a tertiary care hospital. Am J Infect Control. 2010;38:546-51.

2. Wisplinghoff $H$, Bischoff $T$, Tallent $S M$, Seifert $H$, Wenzel RP, Edmond MB. Nosocomial bloodstream infections in US hospitals: analysis of 24,179 cases from a prospective nationwide surveillance study. Clin Infect Dis. 2004;39: 309-17. Erratum in: 2005,40:1077

3. Doi AM, Pignatari ACC, Edmond MB, Marra AR, Camargo LFA, Siqueira RA da Mota VP, Colombo AL. Epidemiology and microbiologic characterization of nosocomial Candidemia from a Brazilian National Surveillance Program. PLoS One. 2016;11:1. 
4. Colombo AL, Ngai AL, Bourque M, Bradshaw SK, Strohmaier KM, Taylor AF, et al. Caspofungin use in patients with invasive candidiasis caused by common non-albicans Candida species: review of the Caspofungin database. Antimicrob Agents Chemother. 2010;54:1864-71.

5. Arendrup MC, Dzajic E, Jensen RH, Johansen HK, Kjaeldgaard P, Knudsen JD, et al. Epidemiological changes with potential implication for antifungal prescription recommendations for fungaemia: data from a nationwide fungaemia surveillance programme. Clin Microbiol Infect. 2013;19:343-53.

6. Colombo AL, Nucci M, Park BJ, Nouer SA, Arthington-Skaggs B, da Matta DA, Warnock D, Morgan J. Brazilian network Candidemia S: epidemiology of candidemia in Brazil: a nationwide sentinel surveillance of candidemia in eleven medical centers. J Clin Microbiol. 2006;44:2816-23.

7. Garnacho-Montero J, Diaz-Martin A, Garcia-Cabrera E, de Pipaon MRP, Hernandez-Caballero C, Lepe-Jimenez JA. Impact on hospital mortality of catheter removal and adequate antifungal therapy in Candida spp. bloodstream infections. J Antimicrob Chemother. 2013;68:206-13.

8. Ibrahim EH, Sherman G, Ward S, Fraser VJ, Kollef MH. The influence of inadequate antimicrobial treatment of bloodstream infections on patient outcomes in the ICU setting. Chest. 2000;118:146-55.

9. Kollef $\mathrm{MH}$. Inadequate antimicrobial treatment: an important determinant of outcome for hospitalized patients. Clin Infect Dis. 2000;31:131-8.

10. Morrell M, Fraser VJ, Kollef MH. Delaying the empiric treatment of Candida bloodstream infection until positive blood culture results are obtained: a potential risk factor for hospital mortality. Antimicrob Agents Chemother. 2005:49:3640-5.

11. Garey KW, Rege M, Pai MP, Mingo DE, Suda KJ, Turpin RS, Bearden DT. Time to initiation of fluconazole therapy impacts mortality in patients with candidemia: a multi-institutional study. Clin Infect Dis. 2006;43:25-31.

12. Bassetti M, Righi E, Ansaldi F, Merelli M, Cecilia T, De Pascale G, Diaz-Martin A, Luzzati R, Rosin C, Lagunes $L$, et al. A multicenter study of septic shock due to candidemia: outcomes and predictors of mortality. Intensive Care Med. 2014:40:839-45.

13. Kludze-Forson M, Eschenauer GA, Kubin CJ, Della-Latta P, Lam SW. The impact of delaying the initiation of appropriate antifungal treatment for Candida bloodstream infection. Med Mycol. 2010;48:436-9.

14. Neoh CF, Liew D, Slavin M, Marriott D, Chen SCA, Morrissey O, et al. Costeffectiveness analysis of anidulafungin versus fluconazole for the treatment of invasive candidiasis. J Antimicrob Chemother. 2011;66:1906-15.

15. Auzinger G, Playford EG, Graham CN, Knox HN, Weinstein D, Kantecki M, et al. Cost-effectiveness analysis of anidulafungin for the treatment of candidaemia and other forms of invasive candidiasis. BMC Infect Dis. 2015;15:463.

16. Pappas PG, Kauffman CA, Andes DR, Clancy CJ, Marr KA, Ostrosky-Zeichner L, Reboli AC, Schuster MG, Vazquez JA, Walsh TJ, et al. Clinical practice guideline for the Management of Candidiasis: 2016 update by the Infectious Diseases Society of America. Clin Infect Dis. 2016;62:409-17.

17. Guillot J, Lebel D, Roy H, Ovetchkine P, Bussieres JF. Usefulness of defined daily dose and days of therapy in pediatrics and obstetrics-gynecology: a comparative analysis of antifungal drugs (2000-2001, 2005-2006, and 2010-2011). J Pediatr Pharmacol Ther. 2014;19:196-201.

18. Kuster SP, Ruef C, Ledergerber B, Hintermann A, Deplazes C, Neuber L, et al. Quantitative antibiotic use in hospitals: comparison of measurements, literature review, and recommendations for a standard of reporting. Infection. 2008;36:549-59.

19. Morris AM, Brener S, Dresser L, Daneman N, Dellit TH, Avdic E, et al. Use of a structured panel process to define quality metrics for antimicrobial stewardship programs. Infect Control Hosp Epidemiol. 2012;33:500-6.

20. Barlam TF, Cosgrove SE, Abbo LM, MacDougall C, Schuetz AN, Septimus EJ, et al. Implementing an antibiotic stewardship program: guidelines by the Infectious Diseases Society of America and the Society for Healthcare Epidemiology of America. Clin Infect Dis. 2016;62:1197-202.

21. Ibrahim OM, Polk RE. Antimicrobial use metrics and benchmarking to improve stewardship outcomes methodology, opportunities, and challenges. Infect Dis Clin N Am. 2014;28:195.

22. World Health Organization. Collaborating Centre for Drug Statistics Methodology [Anatomical therapeutic chemical (ATC) Classification and defined daily doses (DDD)] http://www.whocc.no/atc_ddd_index Accessed 20 Jan 2017.

23. Polk RE, Fox C, Mahoney A, Letcavage J, MacDougall C. Measurement of adult antibacterial drug use in 130 US hospitals: comparison of defined daily dose and days of therapy. Clin Infect Dis. 2007;44:664-70
24. Zagorski BM, Trick WE, Schwartz DN, Wisniewski MF, Hershow RC, Fridkin SK, et al. The effect of renal dysfunction on antimicrobial use measurements. Clin Infect Dis. 2002;35:1491-7.

25. Public Health Ontario. Antimicrobial Stewardship: Metrics and evaluation 2014. http://www.publichealthontario.ca/ Accessed 16 Jan 2017.

26. Puig-Asensio M, Fernandez-Ruiz M, Aguado JM, Merino P, Lora-Pablos D, Guinea J, et al. Propensity score analysis of the role of initial antifungal therapy in the outcome of Candida glabrata bloodstream infections. Antimicrob Agents Chemother. 2016;60:3291-300

27. Nucci M, Colombo AL. Risk factors for breakthrough candidemia. Eur J Clin Microbiol Infect Dis. 2002;21:209-11.

28. Savage RD, Fowler RA, Rishu AH, Bagshaw SM, Cook D, Dodek P, et al. The effect of inadequate initial empiric antimicrobial treatment on mortality in critically ill patients with bloodstream infections: a multi-Centre retrospective cohort study. PLoS One. 2016;11:5

29. Das I, Nightingale P, Patel M, Jumaa P. Epidemiology, clinical characteristics, and outcome of candidemia: experience in a tertiary referral center in the UK. Int J Infect Dis. 2011;15:759-63.

30. Colombo AL, Guimaraes T, Camargo LFA, Richtmann R, de Queiroz-Telles F, Salles MJC, et al. Brazilian guidelines for the management of candidiasis - a joint meeting report of three medical societies: Sociedade Brasileira de Infectologia, Sociedade Paulista de Infectologia and Sociedade Brasileira de Medicina tropical. Braz J Infect Dis. 2013;17:283-312.

31. Clinical and Laboratory Standards Institute. Reference method for broth dilution antifungal susceptibility testing of yeasts: approved standard. In: CLSI document M27-A3. 3rd ed. Wayne, PA, USA: CLSI; 2008.

32. Clinical and Laboratory Standards Institute. Reference method for broth dilution antifungal susceptibility testing of yeasts: fourth informational supplement. CLSI document M27-S4. Wayne, PA, USA: CLSI; 2012. p. 33.

33. Steel RG. Some rank sum multiple comparisons tests. Biometrics. 1961;17:539-52.

34. Fondevilla E, Grau S, Mojal S, Palomar M, Matas L, Gudiol F, Grp VI. Consumption of systemic antifungal agents among acute care hospitals in Catalonia (Spain), 2008-2013. Expert Rev Anti-Infect Ther. 2016;14:137-44.

35. Olaechea-Astigarraga PM, Alvarez-Lerma F, Palomar-Martinez M, InsaustiOrdenana J, Lopez-Pueyo MJ, Seijas-Betolaza I, Otal-Entraigas JJ, GimenoCosta R, Gracia-Arnillas MP, Grp E-H. Trends in systemic antifungal use in critically ill patients. Multicenter observational study, 2006-2010. Enfermedades Infecciosas Y Microbiologia Clinica. 2012;30:435-40.

36. de Souza MCP, dos Santos AG, Reis AMM. Drug utilization study of systemic antifungal agents in a Brazilian tertiary care hospital. Int J Clin Pharm. 2016; 38:1398-406.

37. Gross BN, Steib-Bauert M, Kern WW, Knoth H, Borde JP, Krebs S, et al. Hospital use of systemic antifungal drugs: a multi-center surveillance update from Germany. Infection. 2015;43:423-9.

38. Kd W, Steib-Bauert M, Knoth H, Fr D, Strehl E, Rothe U, et al. Hospital use of systemic antifungal drugs. BMC Clin Pharmacol. 2005;5:1.

39. Zarb P, Amadeo B, Muller A, Drapier N, Vankerckhoven V, Davey P, et al. Antifungal therapy in European hospitals: data from the ESAC pointprevalence surveys 2008 and 2009. Clin Microbiol Infect. 2012;18:389-95.

40. Bansal D, Mangla S, Undela K, Gudala K, D'Cruz S, Sachdev A, Tiwari P. Measurement of adult antimicrobial drug use in tertiary care hospital using defined daily dose and days of therapy. Indian J Pharm Sci. 2014;76:211-7.

41. Bailly S, Maubon D, Fournier P, Pelloux H, Schwebel C, Chapuis C, et al. Impact of antifungal prescription on relative distribution and susceptibility of Candida spp. - trends over 10 years. J Infect. 2016;72:103-11.

42. Colombo AL, Garnica M, Camargo LFA, Da Cunha CA, Bandeira AC, Borghi $D$, et al. Candida glabrata: an emerging pathogen in Brazilian tertiary care hospitals. Med Mycol. 2013;51:38-44.

43. Fournier P, Schwebel C, Maubon D, Vesin A, Lebeau B, Foroni L, et al. Antifungal use influences Candida species distribution and susceptibility in the intensive care unit. J Antimicrob Chemother. 2011;66(12):2880-6.

44. Alexander BD, Johnson MD, Pfeiffer CD, Jiménez-Ortigosa C, Catania J, Booker $\mathrm{R}$, et al. Increasing echinocandin resistance in Candida glabrata: clinical failure correlates with presence of FKS mutations and elevated minimum inhibitory concentrations. Clin Infect Dis. 2013;56(12):1724-32.

45. Villalobos JM, Castro JA, Aviles A, Pelaez MC, Somogyi T, Sandoval L. Candida parapsilosis: a major cause of bloodstream infection in a tertiary care hospital in Costa Rica. Revista Chilena De Infectologia. 2016;33:159-65.

46. Paiva JA, Pereira JM, Tabah A, Mikstacki A, de Carvalho FB, Koulenti D, Ruckly S, Cakar N, Misset B, Dimopoulos G, Antonelli M, Rello J, Ma X, 
Tamowicz B, Timsit JF. Characteristics and risk factors for 28-day mortality of hospital acquired fungemias in ICUs: data from the EUROBACT study. Crit Care. 2016;20:53.

47. Bailly S, Leroy O, Azoulay E, Montravers P, Constantin JM, Dupont H, Guillemot D, Lortholary O, Mira JP, Perrigault PF, et al. Impact of echinocandin on prognosis of proven invasive candidiasis in ICU: a post-hoc causal inference model using the AmarCAND2 study. J Infect. 2017;74:408-17.

48. Murri R, Scoppettuolo G, Ventura G, Fabbiani M, Giovannenze F, Taccari F, et al. Initial antifungal strategy does not correlate with mortality in patients with candidemia. Eur J Clin Microbiol Infect Dis. 2016;35(2):187-93.

Ready to submit your research? Choose BMC and benefit from:

- fast, convenient online submission

- thorough peer review by experienced researchers in your field

- rapid publication on acceptance

- support for research data, including large and complex data types

- gold Open Access which fosters wider collaboration and increased citations

- maximum visibility for your research: over $100 \mathrm{M}$ website views per year 